九州大学学術情報リポジトリ

Kyushu University Institutional Repository

\title{
Elastoplastic Constitutive Equations with Extended Flow Rules
}

Hashiguchi, Koichi

Laboratory of Mechanics for Bio -Production, Faculty of Agriculture, Kyushu University

https://doi.org/10.5109/24059

出版情報：九州大学大学院農学研究院紀要. 38 (3/4)，pp.279-286，1994-03. Kyushu University バージョン：

権利関係 : 


\title{
Elastoplastic Constitutive Equations with Extended Flow Rules
}

\author{
Koichi Hashiguchi \\ Laboratory of Mechanics for Bio -Production, Faculty of Agriculture, \\ Kyushu University 46-05, Fukuoka 812, Japan \\ (Received January 17, 1994)
}

\begin{abstract}
The traditional elastoplastic constitutive equation with a single smooth yield surface is extended so as to describe the dependency of the direction of a plastic stretching on a stress rate or a stretching. Besides, the loading criterion for the extended constitutive equation is formulated. Further, it is shown that a direction of plastic stretching cannot be arbitrary but has to fulfill an inequality under the loading criterion.
\end{abstract}

\section{INTRODUCTION}

A direction of the plastic stretching does not depend on a stress rate in the traditional elastoplastic constitutive equation with a single smooth plastic potential surface. Also, the plastic stretching does not depend on the component tangential to the yield surface but only on the normal component. These dependencies cannot be, however, ignored in the plastic instability phenomenon with a localization of deformation. In order to extend the traditional constitutive equation for the dependency various models have been proposed as follows:

(1) Intersection of yield surfaces (Koiter, 1953; Sewell, 1973, 1974): Plural intersecting yield surfaces are introduced.

(2)Phenomenological comer theory (Hill, 1966; Christoffersen and Hutchinson, 1979) : The existence of a corner in a current stress point on the yield surface is assumed.

(3) $J_{2}$ deformation theory (Rudnicki and Rice, 1975); Storen and Rice, 1975): The plastic stretching due to the stress rate component tangential to the yield surface is introduced. It coincides with the deformation theory of Hencky (1924).

(1) and (2) aim at describing the dependency of the direction of a plastic stretching on a stress rate and predicting a plastic stretching for a range wider than a half of the stress rate space by assuming geometrically a singularity in the field of the outwardnormal of the yield surface, i.e. an existence of a corner in an elastic domain. In fact, however, (1) cannot always describe the dependency of a direction of plastic stretching on a stress rate except for the loading process in which a stress exists continuously on an intersecting point of plural yield surfaces. (2) would not be applicable to the loading process in which a stress rate has a direction of an angle more than 90" from the outward-normal of the central axis of a corner since an evolution rule of the corner has not been clarified for that process up to the present. The mechanical contradiction, i.e. the decrease of a corner height leading to a softening occurs if it is assumed that a tip of the corner follows a stress point in order to describe continuously the dependency of a direction of plastic stretching on a stress rate. It would be difficult or impossible, however, to extend (2) so as to be applicable to the general loading process including a reverse loading and a reloading in various directions, though it has been attempted by 
Tomita et al. (1986) and Goya and Itoh (1991) incorporating a kinematic hardening. (3) is limited to the application to the neighborhood of proportional loading, since it falls within the framework of hypoelasticity relating a stress rate to a stretching linearly although a stress rate - stretching relation has to be nonlinear for the irreversible deformation.

In this article the traditional elastoplastic constitutive equation is extended so as to describe the dependency of the direction of a plastic stretching on a stress rate or a stretching, keeping a single smooth yield surface. Besides, the loading criterion for the extended constitutive equation is formulated, which results in the one proposed a priori by Hill $(1958,1967)$. Further, it is shown that a direction of plastic stretching cannot be arbitrary but has to fulfill an inequality under the loading criterion.

\section{LOADING CRITERION}

A traditional elastoplastic constitutive equation premises on the additive decomposition of the stretching $\boldsymbol{D}$ into the elastic stretching $\boldsymbol{D}^{e}$ and the plastic stretching $\boldsymbol{D}^{\phi}$, i. e.

$$
\boldsymbol{D}=\boldsymbol{D}^{\boldsymbol{e}}+\boldsymbol{D}^{p}
$$

and the elastic stretching is given by

$$
\boldsymbol{D}^{e}=\boldsymbol{E}^{-1} \stackrel{\circ}{\boldsymbol{\sigma}}, \stackrel{\circ}{\boldsymbol{\sigma}}=\boldsymbol{E} \boldsymbol{D}^{e},
$$

where $E$ is the elastic modulus, $\sigma$ is a stress and $\left({ }^{\circ}\right)$ denotes a proper corotational rate. Further, it premises on the existence of the two different processes, i.e. the plastic loading process in which a plastic deformation is induced and the plastic unloading process in which only an elastic deformation is induced. Thus, it can be written that

$$
\begin{array}{r}
\text { plastic loading: } \boldsymbol{D}^{p} \neq \boldsymbol{O}, \\
\text { plastic unloading: } \boldsymbol{D}^{p}=\boldsymbol{O} .
\end{array}
$$

Therefore, the judgment as to which process of them will occur is required when an elastoplastic constitutive equation is applied to the deformation analysis. A standard for the judgement is called a loading criterion. Let the loading criterion be formulated in the following.

Introduce the following assumptions:

Assumption I: A stress rate always induces an elastic stretching so that the elastic modulus $E$ exists, which is uniquely determined for a current state of stress and plastic internal state variables.

Then, the rigid-plastic body is excluded.

Assumption II: The yield surface is regular (smooth).

Therefore, the outward normal of the yield surface is uniquely determined on a point of the yield surface. Constitutive models assuming a corner on the yield surface, e.g. the phenomenological corner theory (Christoffersen and Hutchinson, 1979) are excluded.

Assumption III: The stretching space is divided completely into two half subspaces, i.e. the half subspace for the plastic loading and the one for the plastic unloading in the state that a yield condition is fulfilled. 
This assumption is introduced by Hill (1967). It is the generalization of the fact observed in a uniaxial loading for instance and leads to the irreversibility $\dot{\boldsymbol{\sigma}}^{\prime} \neq-\stackrel{\circ}{\boldsymbol{\sigma}}$ for $\boldsymbol{D}^{\prime}=-\boldsymbol{D}$, where $\dot{\boldsymbol{\sigma}}^{\prime}$ and $\stackrel{\circ}{\boldsymbol{\sigma}}$ are the stress rate induced by the stretchings $\boldsymbol{D}^{\prime}$ and $\boldsymbol{D}$, respectively.

Assumption IV: The stretching for the plastic unloading induces a stress rate directed towards the inside of the yield surface.

Therefore, it holds that

$$
\left.\boldsymbol{D}^{p}=0: \operatorname{tr}(N E D)=\operatorname{tr}(\mathrm{NED})\right)=\operatorname{tr}(\boldsymbol{N} \stackrel{\circ}{ }) \leq 0 .
$$

where tr () stands for a trace and $\mathrm{N}$ is the normalized outward normal of the yield surface, i. e.

$$
N \equiv \frac{\partial f}{\partial \boldsymbol{\sigma}} /\left\|\frac{\partial f}{\partial \boldsymbol{\sigma}}\right\|,
$$

where the yield condition is described as

$$
f\left(\boldsymbol{\sigma}, \boldsymbol{H}_{i}\right)=0,
$$

$\boldsymbol{H}_{i}(i=1,2, \cdots, \mathrm{n})$ denoting collectively scalar - or tensor - valued plastic internal state variables and \|\| designating a magnitude.

By the assumptions I and II the stretching space is divided into two half subspaces by the sign of the quantity tr $(N E D)$, since its sign changes for the stretching with an opposite direction by the relation $\operatorname{tr}\{\boldsymbol{N E}(-\boldsymbol{D})\}=-\operatorname{tr}(N E D)$. Eqn (4) means that the plastic unloading induces a negative sign of $\operatorname{tr}(N E D)$. On account of these facts with the assumption III, it results that the plastic loading and the plastic unloading correspond to $\operatorname{tr}(\boldsymbol{N E D})>\mathbf{0}$ and $\operatorname{tr}(N E D) \leq 0$, respectively. Eventually, the following loading criterion holds.

$$
\left.\begin{array}{l}
\boldsymbol{D}^{p} \neq 0: \operatorname{tr}(N E D)>0 \text { andf }(\boldsymbol{\sigma}, H i)=0 \\
\boldsymbol{D}^{\phi}=0: \operatorname{tr}(\boldsymbol{N E D}) \leq 0 \text { or } f\left(\boldsymbol{\sigma}, \boldsymbol{H}_{i}\right)<0
\end{array}\right\}
$$

In what follows, consider a requirement for a direction of plastic stretching under the loading criterion (7).

Assume the generalized flow rule

$$
\boldsymbol{D}^{p}=\langle\lambda \gg \boldsymbol{P},
$$

where $\lambda$ is a proportionality factor, a function of a stress, plastic internal state variables and a stress rate or a stretching in degree one, and $P$ is a function of a stress, plastic internal state variables and a stress rate or a stretching in degree zero, i.e.

$$
\begin{gathered}
\lambda=\lambda\left(\boldsymbol{\sigma}, \stackrel{\circ}{\boldsymbol{\sigma}}, \boldsymbol{H}_{i}\right) \text { or } \lambda\left(\boldsymbol{\sigma}, D, \boldsymbol{H}_{i}\right), \\
P=P\left(\boldsymbol{\sigma}, \dot{\boldsymbol{\sigma}}, \boldsymbol{H}_{i}\right) \text { or } P(\boldsymbol{\sigma}, D, H i),
\end{gathered}
$$

The bracket $《 \Downarrow$ designates $\langle a\rangle=a$ for $\operatorname{tr}(N E D)>0$ and $f\left(\boldsymbol{\sigma}, \boldsymbol{H}_{i}\right)=0$ and $\langle a\rangle=0$ for $\operatorname{tr}(N E D) \leq 0$ or $f\left(\boldsymbol{\sigma}, \boldsymbol{H}_{i}\right)<0$, where $a$ is an arbitrary scalar variable.

As was described in the previous paper (Hashiguchi, 1993a), the substitution of eqn (8) into the consistency condition of yield condition leads to 


$$
\begin{gathered}
\boldsymbol{D}^{\phi}=\left\langle\frac{\operatorname{tr}(\boldsymbol{N} \dot{\boldsymbol{\sigma}})}{\bar{D}}-\gg \boldsymbol{P},\right. \\
D \equiv-\sum_{i=1}^{\mathrm{n}} \operatorname{tr}\left(\frac{\partial^{\prime} f}{\partial \boldsymbol{H}_{i}} \boldsymbol{h}_{i}\right) /\left\|\frac{\partial f}{\partial \boldsymbol{\sigma}}\right\|,
\end{gathered}
$$

where $\boldsymbol{h}_{i}$ is a function of stress, plastic internal state variables and $\mathrm{P}$ in degree one, which is related to $\stackrel{\circ}{\boldsymbol{H}}_{i}$ as

$$
\stackrel{\circ}{\boldsymbol{H}}_{i}=\ll \lambda \gg \boldsymbol{h}_{i}
$$

since $\stackrel{\circ}{\boldsymbol{H}}_{i}$ includes $\langle\lambda\rangle$ in degree one. $D$ is called the plastic modulus, a function of a stress and plastic internal state variables and a stress rate or a stretching in degree zero, while it is positive, zero and negative for the hardening, the perfectly-plastic and the softening behavior, respectively, which are defined as the deformation behavior that a plastic stretching is induced when a stress rate is directed towards the outside, the tangent and the inside, respectively, of the yield surface, i.e.

$$
\left.\begin{array}{r}
\text { hardening: } \operatorname{tr}(\boldsymbol{N} \stackrel{\circ}{\boldsymbol{\sigma}})>0, \\
\text { perfectly-plastic: } \operatorname{tr}(\boldsymbol{N} \dot{\boldsymbol{\sigma}})=0, \\
\text { softening: } \operatorname{tr}(\boldsymbol{N} \dot{\boldsymbol{\sigma}})<0,
\end{array}\right\}
$$

If the associated flow rule $\boldsymbol{P}=\boldsymbol{N}$ holds, eqn (14) can be rewritten as

$$
\begin{aligned}
& \text { hardening: } \operatorname{tr}\left(\stackrel{\circ}{\boldsymbol{\sigma}} \boldsymbol{D}^{p}\right)>0 \text {, } \\
& \text { perfectly-plastic: } \left.\operatorname{tr}\left(\stackrel{\circ}{\circ} D^{p}\right)=0,\right\} \\
& \text { softening: } \operatorname{tr}\left(\stackrel{\circ}{D^{p}}\right)<0
\end{aligned}
$$

in terms of the stability condition in the small proposed by Drucker (1964).

Eqns (1), (2) and (11) lead to

$$
\boldsymbol{D}=\boldsymbol{E}^{-1} \stackrel{\circ}{\boldsymbol{\sigma}}+\ll \frac{\operatorname{tr}(\boldsymbol{N} \dot{\boldsymbol{\sigma}})}{D} \gg \boldsymbol{P}
$$

Eqn (11) is rewritten by eqns (1) and (2) as

$$
D^{p}=\ll \frac{\operatorname{tr}(N E D)}{D+\operatorname{tr}(N E P)}-\gg P .
$$

Eqns (1), (2) and (17) lead to

$$
\stackrel{\circ}{\boldsymbol{\sigma}}=\boldsymbol{E D}-\boldsymbol{E} \ll \frac{\operatorname{tr}(\boldsymbol{N E D})}{D^{+} \operatorname{tr}(\boldsymbol{N E P})} \gg \boldsymbol{P} .
$$

Obviously, eqn (18) fulfills the continuity condition in the small (Hashiguchi, 1993b) at the boundary between the plastic loading and unloading processes at which $\operatorname{tr}(\boldsymbol{N E D})=0$ holds. The quantity in the bracket $\ll 》$ of eqn (17) has to be positive for the loading process. Thus, $\boldsymbol{P}$ cannot be arbitrary but is required to fulfill the inequality

$$
D+\operatorname{tr}(\boldsymbol{N E P})>\mathbf{0} \text { for } \operatorname{tr}(\boldsymbol{N E D})>\mathbf{0} .
$$

Further, $\boldsymbol{P}$ has to fulfill the inequality

$$
\operatorname{tr}(\boldsymbol{P E D})>0 \text { for } \operatorname{tr}(\boldsymbol{N E D})>0 .
$$


by the work rate-stiffness relaxation (Hashiguchi, 1993a)

$$
\operatorname{tr}\left(D^{p} E D\right) \geq 0
$$

which is one of the fundamental requirements for elastoplastic constitutive equations. The deformation process in the stability in the small $\operatorname{tr}\left(\dot{\sigma}^{D^{p}}\right) \geqq 0$ fulfills the inequality (21) because of the relation

$$
\operatorname{tr}\left(\boldsymbol{D}^{p} \boldsymbol{E D}\right)=\operatorname{tr}\left(\boldsymbol{D}^{p} \boldsymbol{E} \boldsymbol{D}^{p}\right)+\operatorname{tr}\left(\stackrel{\circ}{\boldsymbol{\sigma}} \boldsymbol{D}^{p}\right)
$$

\section{EXTENDED FLOW RULES}

Four types of extended flow rules are proposed in this section.

a) One may assume the following concrete equation for $\mathrm{P}$.

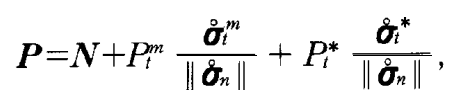

where $P_{t}^{m}$ and $P_{t}^{*}$ are material parameters, and

$$
\begin{aligned}
& \left.\begin{array}{l}
\boldsymbol{\boldsymbol { \sigma }}_{t} \equiv \stackrel{\circ}{\boldsymbol{\sigma}}-\boldsymbol{\sigma}_{n}=\dot{\boldsymbol{\sigma}}_{t}^{\mathrm{m}}+\stackrel{\circ}{\boldsymbol{\sigma}}^{*}, \\
\boldsymbol{\sigma}_{n} \equiv \operatorname{tr}(\boldsymbol{N} \boldsymbol{\sigma}) \boldsymbol{N},
\end{array}\right\} \\
& \begin{array}{c}
\dot{\boldsymbol{\sigma}}_{t}^{m} \equiv \dot{\boldsymbol{\sigma}}^{m}-\dot{\boldsymbol{\sigma}}_{n}^{m}=\dot{\boldsymbol{\sigma}}_{m}\{\boldsymbol{I}-(\operatorname{tr} \boldsymbol{N}) \boldsymbol{N}\}, \\
\dot{\boldsymbol{\sigma}}_{t}^{*} \equiv \boldsymbol{\sigma}-\boldsymbol{\sigma}_{n}^{*},
\end{array} \\
& \left.\begin{array}{l}
\dot{\boldsymbol{\sigma}}_{n}^{m} \equiv \operatorname{tr}\left(\boldsymbol{N} \dot{\boldsymbol{\sigma}}^{m}\right) \boldsymbol{N}=\dot{\boldsymbol{\sigma}}_{m}(\operatorname{tr} \boldsymbol{N}) \boldsymbol{N}, \\
\dot{\boldsymbol{\sigma}}_{n}^{*} \equiv \operatorname{tr}\left(\boldsymbol{N} \dot{\boldsymbol{\sigma}}^{*}\right) \boldsymbol{N},
\end{array}\right\} \\
& \left.\begin{array}{l}
\dot{\sigma}_{m} \equiv \frac{1}{3} \operatorname{tr} \stackrel{\circ}{\boldsymbol{\sigma}}, \dot{\boldsymbol{\sigma}}_{m} \equiv \dot{\boldsymbol{\sigma}}_{m} \boldsymbol{I}, \\
\dot{\boldsymbol{\sigma}}^{*} \equiv \dot{\boldsymbol{\sigma}}-\dot{\boldsymbol{\sigma}}_{m} .
\end{array}\right\}
\end{aligned}
$$

( ) stands for a material -time derivative. Eqn (23) is the slight modification of the one for the tangential plasticity proposed by the author (Hashiguchi, 1993a). $P_{t}^{m}$ is set as $P_{t}^{m}=0$ for plastically-incompressible materials.

Let the plastically-incompressible and hardening material with $P_{t}^{m}=0, \operatorname{tr} \boldsymbol{N}=0$ and $\operatorname{tr}(\boldsymbol{N} \dot{\boldsymbol{\sigma}}) \geq 0$ be considered. It fulfills the work rate-stiffness relaxation by eqn (22) and the relation

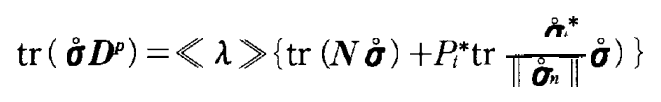

with the inequality

$$
\operatorname{tr}\left(\stackrel{\circ}{\boldsymbol{\sigma}}_{t}^{*} \stackrel{\circ}{\boldsymbol{\sigma}}\right)=\operatorname{tr}\left[\left\{\stackrel{\circ}{\boldsymbol{\sigma}}^{*}-\operatorname{tr}\left(\boldsymbol{N} \dot{\circ}^{*}\right) \boldsymbol{N}\right\} \stackrel{\circ}{\boldsymbol{\sigma}}\right]=\left\|\stackrel{\circ}{\boldsymbol{\sigma}}^{*}\right\|^{2}\left\{\boldsymbol{I}-\operatorname{tr}^{2}\left(\boldsymbol{N} \stackrel{\circ}{\boldsymbol{\sigma}}^{*}\right)\right\} \geq 0 .
$$

Next consider the plastically-incompressible material with $P_{t}^{m}=0, \operatorname{tr} \boldsymbol{N}=0$ and the elastic modulus of Hooke's type

$$
E_{i j k l}=L \delta_{i j} \delta_{k i}+2 G \delta_{i k} \delta_{i l}
$$

where $L$ and $G$ are the Lame's constant and the elastic shear modulus, respectively, and 
$\delta_{i j}$ is the Kronecker's delta.

The work rate-stiffness relaxation is fulfilled by the relation

$$
\operatorname{tr}\left(\boldsymbol{D}^{p} \boldsymbol{E D}\right)=\ll \lambda \gg\left\{\operatorname{tr}(\mathrm{NED})+P_{t}^{*} \operatorname{tr}\left(\frac{\dot{\circ}_{t}^{*}}{\left\|\dot{\boldsymbol{\sigma}}_{n}\right\|} \boldsymbol{E D}\right)\right\}
$$

with the inequality

$$
\begin{aligned}
\operatorname{tr}\left(\stackrel{\circ}{\boldsymbol{\sigma}}_{t}^{*} \boldsymbol{E} \boldsymbol{D}\right) & =\left(\stackrel{\circ}{\boldsymbol{\sigma}}_{t}^{*}\right)_{i j}\left(\boldsymbol{L} \boldsymbol{\delta}_{i j} \boldsymbol{\delta}_{k l} D_{k l}+2 G D_{i j}\right) \\
& =2 G \operatorname{tr}\left(\dot{\boldsymbol{\sigma}}_{t}^{*} \boldsymbol{D}\right) \\
& =2 G\left[\operatorname{tr}\left(\dot{\circ}_{t}^{*} \boldsymbol{D}^{e}\right)+\ll \lambda \gg\left\{\operatorname{tr}\left(\boldsymbol{N} \dot{\boldsymbol{\sigma}}_{t}^{*}\right)+P \frac{\left\|\dot{\boldsymbol{\sigma}}_{t}^{*}\right\|^{2}}{\left\|\stackrel{\circ}{\boldsymbol{\sigma}}_{n}\right\|}\right\}\right] \\
& \geq 2 G \operatorname{tr}\left(\stackrel{\circ}{\boldsymbol{\sigma}}_{t}^{*} \boldsymbol{D}^{e}\right) \\
& =2 G \operatorname{tr}\left[\left\{\dot{\boldsymbol{\sigma}}^{*}-\operatorname{tr}\left(\boldsymbol{N} \dot{\boldsymbol{\sigma}}^{*}\right) \boldsymbol{N}\right\} \boldsymbol{D}^{e}\right] \\
& =(2 G)^{2}\left\{\operatorname{tr}\left(\boldsymbol{D}^{e *} \boldsymbol{D}^{e}\right)-\operatorname{tr}\left(\boldsymbol{N} \boldsymbol{D}^{e *}\right) \operatorname{tr}\left(\boldsymbol{N} \boldsymbol{D}^{e}\right)\right\} \\
& =\left(2 G\left\|\boldsymbol{D}^{e *}\right\|\right)^{2}\left\{1-\operatorname{tr}^{2}\left(\boldsymbol{N} \frac{\boldsymbol{D}^{e *}}{\left\|\boldsymbol{D}^{e *}\right\|}\right)\right\} \\
& \geq 0
\end{aligned}
$$

noting the relation $\operatorname{tr}\left(\boldsymbol{N} \dot{\boldsymbol{\sigma}}_{t}{ }^{*}\right)=0$ and $\operatorname{tr}\left(\boldsymbol{N} \boldsymbol{D}^{e *}\right)=\operatorname{tr}(\mathrm{ND} ")$.

b)A simpler flow rule is

$$
\boldsymbol{P}=\boldsymbol{N}\left(\boldsymbol{I}+C_{t} \frac{\stackrel{\circ}{\boldsymbol{\sigma}}_{t}}{\left\|\stackrel{\circ}{\boldsymbol{\sigma}}_{n}\right\|}\right),
$$

where $C_{t}$ is a material parameter.

The tangential component in eqn (33) with $\operatorname{tr} \mathrm{N}=\mathrm{O}$ does not bring about a plastic volume change, i. e.

$$
\operatorname{tr} P=0
$$

noting $\operatorname{tr}\left(\boldsymbol{N} \stackrel{\circ}{\boldsymbol{\sigma}}_{t}\right)=0$.

c) A flow rule different slightly from eqn (33) is

$$
\boldsymbol{P}=\boldsymbol{N}\left(\boldsymbol{I}+A_{t} \mid \frac{\stackrel{\circ}{\sigma}_{t}}{\boldsymbol{N} \stackrel{\circ}{\boldsymbol{\sigma}} \|}\right),
$$

where $A_{t}$ is a material parameter. Needless to say, the flow rule (35) also fulfills eqn (34).

A further consideration is required as to the fulfillment of the work rate -stiffness relaxation for the flow rules (33) and (35).

d) A flow rule with a stretching is

$$
\boldsymbol{P}=\boldsymbol{N}+D_{t} \frac{\boldsymbol{D}}{\|\boldsymbol{D}\|},
$$

where $D_{t}$ is a material parameter.

The flow rule (36) always fulfills the work rate-stiffness relaxation since it holds that

$$
\operatorname{tr}\left(\boldsymbol{D}^{p} \boldsymbol{E} \boldsymbol{D}\right)=\left\langle\lambda \gg\left\{\operatorname{tr}(\mathrm{NED})+C_{t} \operatorname{tr}(\boldsymbol{D E} \boldsymbol{D}) / \| \boldsymbol{D} \text { II }\right\} \quad \geq 0,\right.
$$

provided that the elastic modulus $\boldsymbol{E}$ is a positive-definite tensor. 
e)A special case of the flow rule (36) is given as

$$
\boldsymbol{P}=\boldsymbol{N}+D_{t}^{v} \frac{D_{v}}{\|\boldsymbol{D}\|} I+D_{t}^{*} \frac{D^{*}}{\left\|D^{*}\right\|},
$$

where

$$
D_{v} \equiv \operatorname{tr} \boldsymbol{D}, \boldsymbol{D}^{*} \equiv \boldsymbol{D}-\frac{1}{3} D_{v} \boldsymbol{I} .
$$

$D_{t}^{v}$ and $D_{t}^{*}$ are material parameters. The flow rule (38) with $\operatorname{tr} N=0$ and $D_{t}^{v}=0$ fulfills eqn (34). That is, it does not bring about a plastic volume change.

The flow rule (38) fulfills the work rate -stiffness relaxation for the elastic modulus of Hooke's type (30) because of

$$
\operatorname{tr}\left(\boldsymbol{D}^{p} \boldsymbol{E D}\right)=\left\langle\lambda \gg\left\{\operatorname{tr}(\boldsymbol{N E D})+(3 L+2 G) D_{t}^{v} D_{v}^{2} /\|\boldsymbol{D}\|+2 G D_{t}^{*}\left\|\boldsymbol{D}^{*}\right\|\right\} \geq 0\right.
$$

The plastic moduli $\boldsymbol{D}$ of the flow rules (36) and (38) include a stretching (degree zero) but do not include a stress rate so that a stress rate is expressed by a stretching but inversely a stretching cannot be expressed by a stress rate. The former expression is convenient for the ordinary finite element programming based on the displacement method. On the other hand, the plastic moduli of the flow rules (23), (33) and (35) include a stress rate (degree one) and thus a stress rate cannot be expressed by a stretching and also a stretching cannot be expressed by a stress rate.

\section{REFERENCES}

Christoffersen, J. and Hutchinson, J. W. 1979 A class of phenomenological corner theories of plasticity, J.Mech.Phys solids, 27:465-487.

Drucker, D. C. 1964 On the postulate of stability of material in the mechanics of continua, J.de Mechanique, 3: 235-249.

Goya, M. and Itoh, K. 1991 An expression of elastic-plastic constitutive laws incorporating vertex formulation and kinematic hardening, J.Appl.Mech., 58: 617622.

Hashiguchi, K. 1993a Fundamental requirements and formulation of elastoplastic constitutive equations with tangential plasticity, Int.J. Plasticity, 9: 525-549.

Hashiguchi, K. 1993b Mechanical requirements and structures of cyclic plasticity models, Int. $J$. Plasticity, 9: 721-748.

Hencky, H. 1924 Zur Theorie plastischer Deformation und der hierdurch im Material herforgerufenen Nachspannungen, Z.A. M. M., 4: 323-334.

Hill, R. 1958 A general theory of uniqueness and stability in elastic-plastic solids. J.Mech. Phys. Solids, 6: 236-249.

Hill, R. 1966 Generalized constitutive laws for elastic-plastic solids, J. Mech. Phys. Solids, 14: 95 102 .

Hill, R. 1967 On the classical constitutive relations for elastic/plastic solids, Recent Progress in Appl.Mech., pp. 241-249.

Koiter, W. T. 1953 Stress-strain relations, uniqueness and variational theories for elasticplastic materials with singular yield surfaces, Quart.Appl. Math., 11: 350-354.

Rudnicki, J. W. and Rice, J. R. 1975 Conditions for the localization of deformation in pressure sensitive dilatant materials, J.Mech. Phys. Solids, 23: 371-394.

Sewell, M. J. 1973 A yield-surface corner lowers the buckling stress of an elastic-plastic plate under compression, J.Mech.Phys. Solids, 21: 19-45.

Sewell, M. J. 1974 A plastic flow at a yield vertex, J. Mech. Phys. Solids, 22: 469-490.

Storen, S. and Rice, J. R. 1975 Localized necking in thin sheets,/. Mech. Phys. Solids, 23: 421-441. 
Tomita,Y., Shindoh, A., Kim, Y. S. and Michiura, K. 1986 Deformation behavior of elastic-plastic tubes under extended pressure and axial load, Int. J. Mech. Sci., 20: 263-275. 